\title{
Wong-Type Dermatomyositis Showing Porokeratosis-Like Changes (Columnar Dyskeratosis): A Case Report and Review of the Literature
}

\author{
Nicole Umanoff ${ }^{a}$ Ari Fisher $^{b} \quad$ J. Andrew Carlson ${ }^{a}$ \\ a Divisions of Dermatology and Dermatopathology, Department of Pathology, \\ Albany Medical College, and b Albany Dermatology, Albany, N.Y., USA
}

\author{
Key Words \\ Dermatomyositis · Pityriasis rubra pilaris · Porokeratosis · Wong type · \\ Columnar dyskeratosis
}

\section{Abstract}

Background: Wong-type dermatomyositis (DM) exhibits simultaneous pityriasis rubra pilaris (PRP) features. Case Report: A 50-year-old woman presented with a heliotrope rash, Gottron's papules, and a poikilodermic, erythematous rash in shawl distribution without evidence of muscle weakness. Despite topical corticosteroids, the eruption progressed 9 months later to include generalized hyperkeratotic follicular papules, islands of sparing, and atrophic macules with a collarette of scale suggestive of porokeratosis. Mild dysphonia was the only sign of muscle weakness. Serology showed positive ANA. Histopathology revealed interface dermatitis with dermal mucin and melanophages, irregular psoriasiform hyperplasia, alternating mounds of para- and orthokeratosis, and tiers of dyskeratotic cells (columnar dyskeratosis). Systemic corticosteroid therapy was not tolerated; acitretin diminished the hyperkeratosis. While hyperpigmentation persisted, no progression of cutaneous or muscular symptoms has occurred after 22 months of follow-up and cessation of the therapy. Overall, her course did not differ from the natural history documented in the literature review of Wong-type DM. The most similar case also exhibited pseudocornoid lamella changes. Conclusion: Wong-type DM is a clinicopathologic DM-PRP hybrid that can also exhibit porokeratosis-like features best described as columnar dyskeratosis. Recognizing these types of lesions in DM is warranted in order to make an accurate assessment of their prognostic significance. 


\section{Introduction}

Dermatomyositis (DM) is an inflammatory cutaneous and myopathic condition that classically presents in older patients with heliotrope rash, Gottron's papules, coarse erythroderma of the neck, chest and shoulders, and proximal muscle weakness that can signal the presence of an underlying malignancy in a minority [1]. A unique variant of DM known as Wong-type DM describes the superimposition of the clinical and histopathologic features of pityriasis rubra pilaris (PRP) onto DM. Wong-type DM is named after K.O. Wong, who reported a series of $23 \mathrm{DM}$ patients, 11 of whom had hyperkeratotic follicular papules [2]. Herein, we review the literature [2-15] and report an additional case of Wong-type DM with coexisting clinicopathologic features that mimicked porokeratosis.

\section{Case Report}

A 50-year-old woman with insulin-dependent diabetes presented to the dermatology department with a scaly, itchy rash on her arms and back that spread to her face, scalp, and then to most of her body. She also had a periorbital heliotrope rash and flat, scaly, pink papules on the dorsum of both hands with mild eponychial inflammation. There were no complaints or signs of muscle weakness. Topical corticosteroid therapy was prescribed. Nine months later, her rash progressed with the development of confluent hyperkeratotic patches and papules of itchy, coarse, dirty grayish, dry skin with areas of dull-pink coloration. In addition to generalized hyperkeratosis, scattered hypopigmented macules and papules with a rim of fine scaling on both forearms was apparent. Sharply demarcated skip areas were present around her waist and medial and inferior breasts, as well as on the sides of her hands and feet. The heliotrope rash had resolved, but Gottron's papules had persisted (fig. 1). Dry, coarse skin on the sides of the face and scalp with dyschromia, as well as patchy, nonscarring alopecia, were present. She reported new sequelae of transient 'swelling' of varied areas of the skin, 'stiffness' in her hips, and difficulties 'hitting the high notes' while recreationally singing (dysphonia). She denied any other symptoms such as muscle weakness. On physical examination, the patient was a well-appearing, obese woman in no acute distress. Her vitals were all within normal limits. There was no lymphadenopathy. Her muscle strength was $5 / 5$, her range of motion intact, and there was no joint or muscle pain or tenderness upon palpation. Serology was significant only for positive ANA and mildly elevated glucose. The rest of the chemistry panel, thyroid-stimulating hormone, aldolase, creatine kinase, and complete blood count were normal. In addition to insulin-dependent diabetes with use of the insulin pump, her previous medical history included obesity, hyperlipidemia, hypothyroidism, asthma, and seasonal allergies, managed by an HMG-CoA reductase inhibitor, levothyroxine, albuterol inhaler, and cetirizine, respectively. She had had a carpal tunnel repair many years previously.

\section{Histopathology}

A punch biopsy performed at first presentation showed a subtle, cell-poor, vacuolar interface dermatitis with rare melanophages consistent with DM, and a focus of suppurative folliculitis, the latter of which was interpreted to represent an incidental process. Punch biopsies taken after the rash had progressed showed more severe alterations compared to the first biopsy. Specifically, the dominant changes consisted of lymphocytic vacuolar interface dermatitis with effacement of some rete ridges, clusters of necrotic keratinocytes, and 

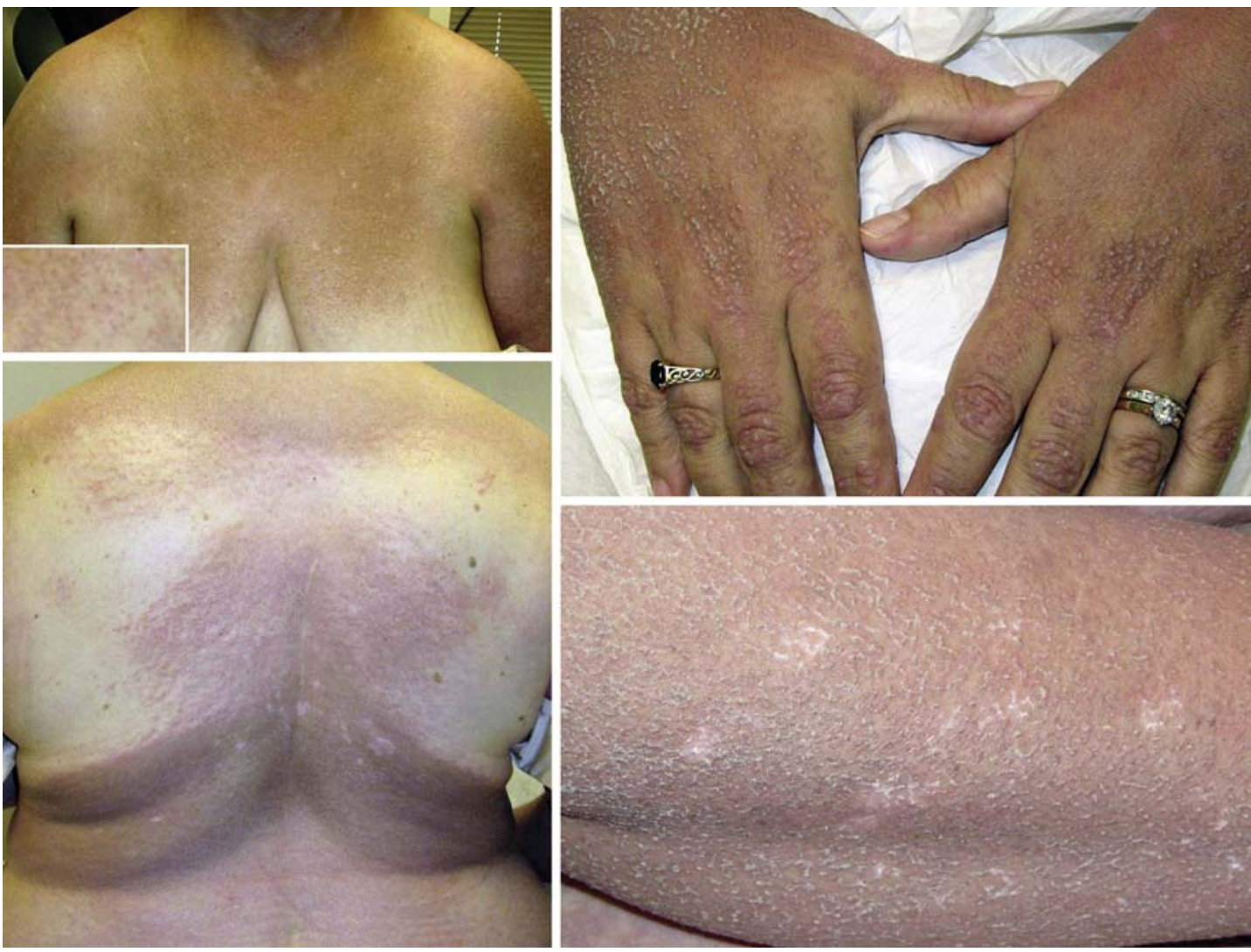

Fig. 1. Clinical presentation of Wong-type DM with porokeratosis-like changes. The top left panel shows a diffuse hyperpigmented plaque and keratotic follicular papules (inset). The bottom left panel highlights confluent patches and plaques of coarse, dirty grayish, hyperkeratotic skin with areas of dull-pink discoloration as well as islands of sparing. The top right panel demonstrates Gottron's papules over the phalangeal-metacarpal and interphalangeal joints as well as numerous markedly keratotic papules over the dorsal hands and forearms. The bottom right panel shows, within the regions of generalized hyperkeratosis, scattered hypopigmented lesions with a collarette of fine scale reminiscent of porokeratosis.

numerous melanophages and free melanin found at the dermal-epidermal junction and papillary dermis. In addition, extensive dermal mucin deposition was evident. In addition, irregular psoriasiform hyperplasia, alternating mounds of para- and orthokeratosis, and follicular plugging were superimposed on the interface alterations. No basement membrane thickening or dermal infiltrates of neutrophils and/or eosinophils were identified (fig. 2). Biopsy of the papules with fine collarettes of scale showed discontinuous vertical tiers of dyskeratotic cells admixed with parakeratotic cells associated with underlying focal hypogranulosis, vacuolar interface alterations and degenerated keratinocytes. This constellation of histopathologic findings resembles those described for columnar dyskeratosis (fig. 3) [16]. Direct immunofluorescence studies demonstrated C3 superficial vascular deposits, trace basement membrane IgM deposits, and numerous colloid bodies - changes that can be found in DM [15]. 
Umanoff et al.: Wong-Type Dermatomyositis Showing Porokeratosis-Like Changes (Columnar Dyskeratosis): A Case Report and Review of the Literature
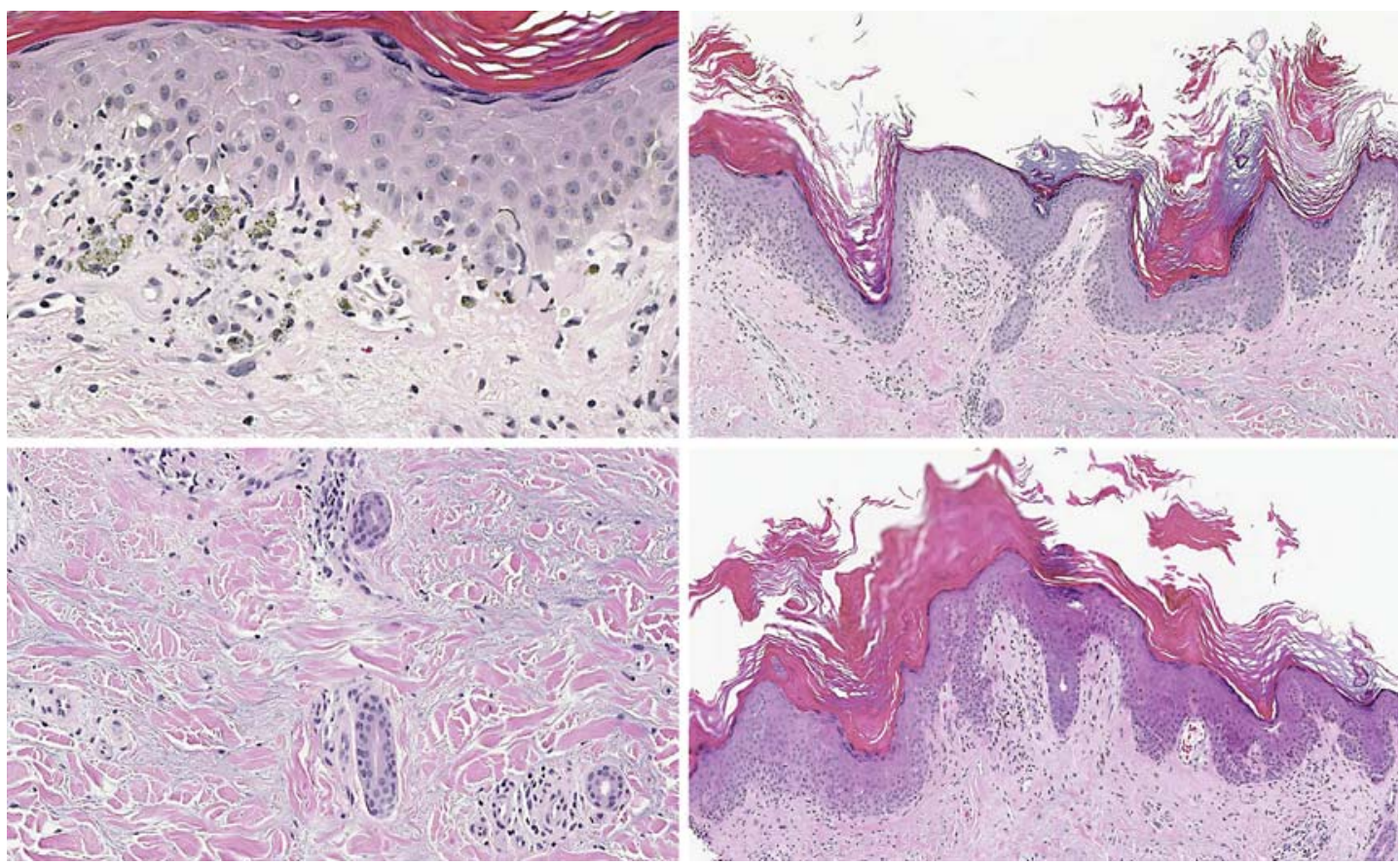

Fig. 2. Concomitant histopathology of DM and PRP. The top left panel shows the characteristic changes of DM consisting of vacuolar interface alterations, numerous melanophages, free melanin in the papillary dermis, cytoid bodies, and necrotic keratinocytes; in the bottom left panel, abundant mucin deposition is visible. The top and bottom right panels show histopathologic findings of PRP with irregular psoriasiform hyperplasia, alternating mounds of para- and orthokeratosis, follicular plugging, and an intact granular layer.

\section{Outcome}

Initial treatment with oral prednisone was discontinued due to exacerbation of her diabetes. In place of corticosteroid therapy, alternating 25/35 mg Soriatane daily resulted in a marked diminishment of the hyperkeratotic papules. However, her skin remained hyperpigmented in the affected regions. After 22 months of follow-up, she is stable without renewed onset of cutaneous or muscular symptoms.

\section{Literature Review}

A systematic review of the literature revealed 25 cases of Wong-type DM since 1956 [2-15]. The average age at presentation was 40 years, with a median age of 46 years and a range from 9 to 89 years. The sex ratio (M:F) was 1:2.5, illustrating that the most commonly affected population is middle-aged women. Two patients had a history of eczema/atopic dermatitis [5, 6], and 1 patient had preexisting chronic myelogenous leukemia in remission [15]. Presenting symptoms included a rash (100\%), muscle weakness (large muscles $92 \%$, dysphagia/dysphonia $>20 \%$ ), and fatigue/flu-like illness (33\%). Signs included an erythematous hyperkeratotic eruption (100\%), heliotrope rash (88\%), Gottron's papules (64\%), and palmar-plantar keratoderma (36\%). The order of onset of PRP was varied, with no pattern significantly dominating: 6 cases developed signs of PRP before DM, 4 cases showed DM before PRP, and 3 cases had a simultaneous onset of PRP and DM. Histopathology showed 

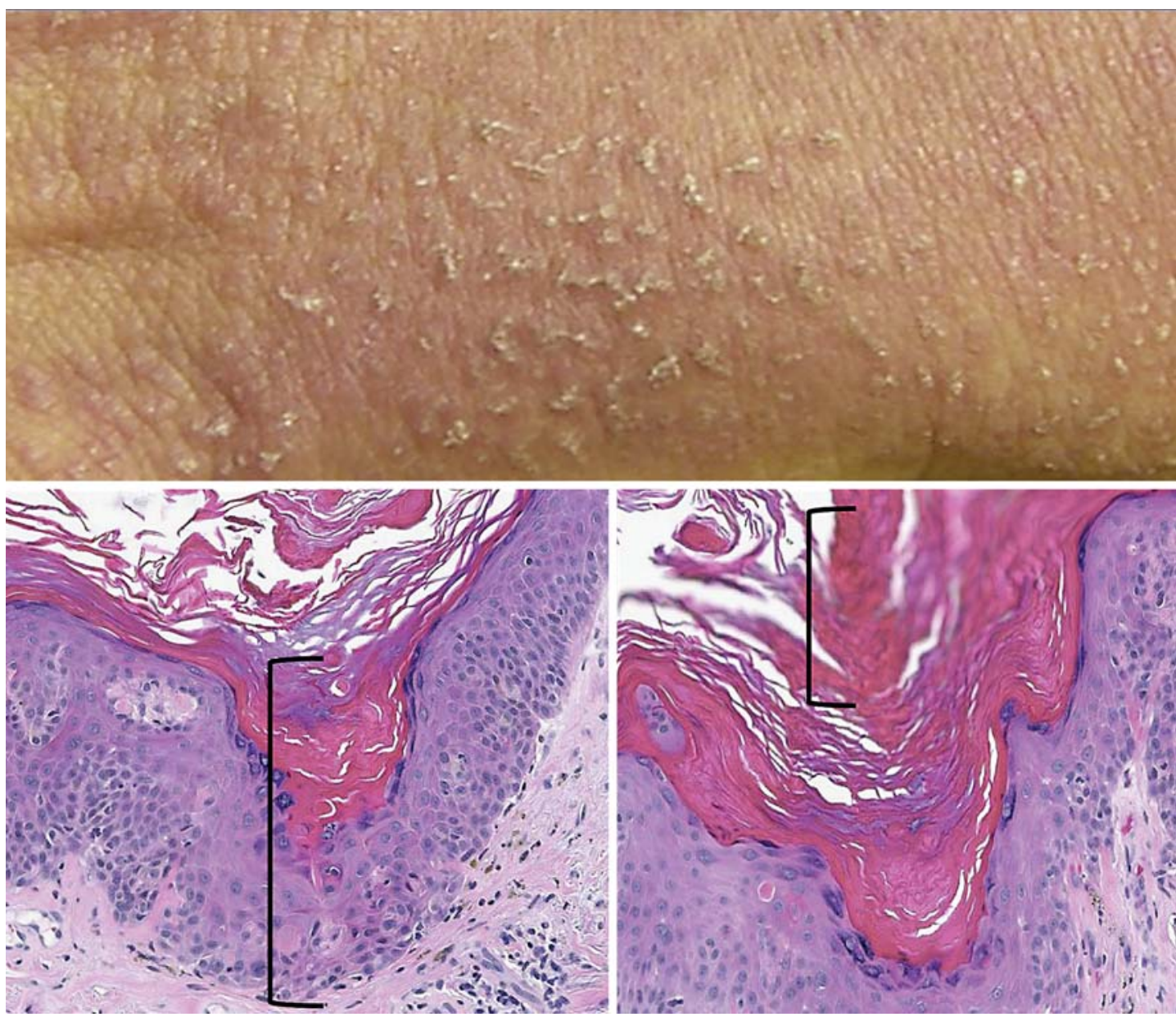

Fig. 3. Pseudocornoid lamella (columnar dyskeratosis). The top panel shows numerous, fine collarettes of scale at the edges and overlying keratotic papules suggestive of porokeratosis. However, microscopically, typical cornoid lamellae were not present. The bottom left and right panels show epidermal dells plugged by dyskeratotic (necrotic), parakeratotic, and orthokeratotic keratinocytes arranged vertically rather than diagonally, as found in typical lesions of porokeratosis [19]. At the dermoepidermal junction, focal vacuolar interface changes are present, which are associated with necrotic keratinocytes and colloid bodies. Overlying these regions are discontinuous tiers of mostly dyskeratotic cells (highlighted by the brackets). This constellation of findings best matches a recently described disorder termed 'columnar dyskeratosis' [16].

follicular hyperkeratosis (76\%), vacuolar interface dermatitis (72\%), hyperkeratosis composed of alternating areas of ortho- and parakeratosis arranged in both horizontal and vertical directions (25\%), dermal mucin deposition (16\%), and arrector pili myositis (16\%). No basement membrane thickening or coexisting neutrophilic infiltrate has been described. However, rare eosinophils were found in the lesional skin of a 54-year-old man with Wongtype DM associated with anti-melanoma differentiation-associated gene 5 (anti-MDA5) antibodies [14]. All muscle biopsies performed showed perifascicular atrophy and perivascular inflammation. Serology showed elevated muscle enzymes (creatine phosphokinase > aldolase $>$ LDH, ALT; 91\%), positive ANA (36\%), and autoantibodies (anti-smith, anti-DNA, and antiMDA5; 18\%). When performed, all EMG results were consistent with inflammatory myositis.

Treatment consisted of systemic corticosteroids, antimalarials (hydroquinone), and/or cytotoxic agents (methotrexate, azathioprine, or mycophenolate mofetil), alone or in combi- 
nation: corticosteroids alone (60\%), in combination with immunosuppressant lesions (28\%), or hydroquinone (4\%). No reports of retinoid therapy exist. The outcomes were full resolution of cutaneous and muscular disease (64\%), cutaneous resolution only (7\%), and cutaneous and muscle improvement but not resolution (14\%); the remainder of patients was lost to follow-up (15\%). The average follow-up time was 45 months (range 8-168). In none of the patients, an underlying malignancy was discovered during their disease course.

The literature revealed only 1 other case of Wong-type DM with porokeratosis-like lesions, in a 53-year-old woman reported by Lupton et al. [9]. Other similarities to our case included dysphonia and positive ANA with the absence of any autoantibodies (Scl-70, SS-A, SS-B, anti-Jo1, anti-RNP, anti-smith, and anti-DNA). In contrast, Lupton's case presented with PRP and then developed DM with porokeratosis-like lesions, whereas our patient first presented with signs of DM. Moreover, Lupton's patient suffered systemic symptoms (weight loss, difficulty sleeping), displayed severe muscle weakness, reported at one point the inability to sit upright on her own, and had elevated aldolase and creatine kinase. While our patient had several chronic diseases, there was no apparent correlation to her rash, and after nearly 2 years of follow-up, she still lacked any significant muscle weakness. Lupton described the lesions as 'porokeratosis-like', without providing any clinical photographs. The histology was described as a 'pseudo-cornoid lamella with focal loss of the granular layer with overlying column of parakeratotic cells in delled epidermis' [9]. This photomicrograph also shows dyskeratotic rather than parakeratotic cells, which is similar to the findings in our patient. Lastly, our patient is doing well off of retinoid therapy, whereas the DM in Lupton's patient is maintained on low-dose prednisone and weekly oral methotrexate as she cannot tolerate lower doses.

\section{Discussion}

The dynamic, diverse clinical and histological features described above illuminate the singularity of Wong-type DM. It is a diagnostic challenge if one is not aware of this hybrid disease, which depending on its onset, could be labeled as DM or PRP. Both conditions alone have been associated with underlying malignancy and immunosuppression $[2,17,18]$. Porokeratosis is also associated with decreased immune surveillance and systemic diseases [19]. However, based on the literature review, Wong-type DM lacks an association with malignancy or cause of immune suppression, even if the short duration of follow-up, the relatively low number of reported cases, and the young age at presentation warrants a malignancy work-up. Nonetheless, Wong-type DM resolved in most cases, suggesting a short-lived trigger and/or cause.

The most unique feature seen in this case was the development of several regions of grouped macules and papules showing a collarette of scale that histopathologically corresponded to irregular vertical columns of dyskeratotic cells within epidermal dells. These structures represented pseudocornoid lamellae. A typical cornoid lamella exhibits a diagonally oriented column of tightly fitted parakeratotic cells. The vertical columns of mostly dyskeratotic rather than parakeratotic cells reported herein most closely resemble the recently described, putative keratinization disorder termed 'columnar dyskeratosis' [16]. The 2 patients described in this case report presented with widespread verrucous, red-brown plaques; only one of them demonstrated clinical findings suggestive of porokeratosis: a fine scale on the palms of an organ transplant recipient. The other patient did not use immunosuppressants; thus, an immune surveillance problem was discounted. A drug etiology (from atorvastatin) was considered; however, no reports of a similar eruption have been documented [16]. The fact that 2 patients presented with identical histopathologic findings 
suggests a unique reaction pattern, if not a clinicopathologic entity that is a manifestation of cutaneous stress and the skin's attempt to maintain homeostasis [20]. Overall, columnar dyskeratosis is the best, most apt histopathologic description of this unique pattern of dyskeratosis.

In conclusion, for patients with signs of DM and PRP, the diagnosis of Wong-type DM should be considered, and prompt treatment should be started, with regular follow-up to find an individualized, effective regimen. The addition of porokeratosis-like changes is also an exceptional characteristic of Wong-type DM. The pathogenesis and clinical significance of this particular phenomenon of columnar dyskeratosis requires further elucidation. Therefore, we urge physicians to search for these alterations in patients with DM to determine disease associations and prognostic significance.

\section{Acknowledgments}

This case study was supported in part by clinical revenues and generous donations to the Divisions of Dermatology and Dermatopathology, Department of Pathology, Albany Medical College.

\section{Disclosure Statement}

The authors declare no conflicts of interest.

\section{References}

1 Femia AN, Vleugels RA, Callen JP: Cutaneous dermatomyositis: an updated review of treatment options and internal associations. Am J Clin Dermatol 2013;14:291-313.

$\checkmark 2$ Wong KO: Dermatomyositis: a clinical investigation of twenty-three cases in Hong Kong. Br J Dermatol 1969; 81:544-547.

-3 Dermatomyositis. AMA Arch Derm Syphilol 1953;68:101-102.

4 Christianson HB, Brunsting LA, Perry HO: Dermatomyositis: unusual features, complications, and treatment. AMA Arch Derm 1956;74:581-589.

5 Dupré A, Bonafé JL, Delsol G, Oksman F: Alterations of the arrector pili muscle in a case of dermatomyositis (author's transl) (in French). Dermatologica 1976;152:33-39.

6 Granier H, Bressollette L, Morand C, Gavanou J, Mottier D: Dermatomyositis type Wong in a 50-year-old woman (in French). Ann Med Interne (Paris) 1991;142:389.

7 de la Tribonnière X, Delaporte E, Alfandari S, Rowland Payne CM, Piette F, Bergoend H: Dermatomyositis with follicular hyperkeratosis. Dermatology 1995;191:242-244.

-8 Requena L, Grilli R, Soriano L, Escalonilla P, Farina C, Martin L: Dermatomyositis with a pityriasis rubra pilarislike eruption: a little-known distinctive cutaneous manifestation of dermatomyositis. Br J Dermatol 1997;136: 768-771.

-9 Lupton JR, Figueroa P, Berberian BJ, Sulica VI: An unusual presentation of dermatomyositis: the type Wong variant revisited. J Am Acad Dermatol 2000;43:908-912.

10 Caporali R, Cavagna L, Bellosta M, Bogliolo L, Montecucco C: Inflammatory myopathy in a patient with cutaneous findings of pityriasis rubra pilaris: a case of Wong's dermatomyositis. Clin Rheumatol 2004;23:63-65.

11 Polat M, Lenk N, Ustun H, Oztas P, Artuz F, Alli N: Dermatomyositis with a pityriasis rubra pilaris-like eruption: an uncommon cutaneous manifestation in dermatomyositis. Pediatr Dermatol 2007;24:151-154.

12 Beckum KM, Gavino AC, Marks S, Andea AA, Elewski BE: Wong type dermatomyositis: 20th case reported. J Drugs Dermatol 2010;9:1475-1476.

13 Haro R, Revelles JM, Fariña Mdel C, Martín L, Requena L: Wong's dermatomyositis: a new case and review of the literature. Int J Dermatol 2013;52:466-470.

14 Canavan T, Sidorsky T, Doan LT, Ricardo-Gonzalez RR, Shen G, Rosenblum MD: A case of Wong-type dermatomyositis with concomitant anti-MDA5 features. J Am Acad Dermatol 2014;70:e62-e64. 
15 Magro CM: Dermatomyositis of Wong: a rare dermatomyositis variant. Dermatologist, July 2014, vol 22. http://www.the-dermatologist.com/content/dermatomyositis-wong-rare-dermatomyositis-variant.

16 Pock L, Stork J: Two case reports of columnar dyskeratosis, an unusual keratinisation disorder. Dermatology 2010;220:274-279.

17 Sanchez-Regana M, Lopez-Gil F, Salleras M, Umbert P: Pityriasis rubra pilaris as the initial manifestation of internal neoplasia. Clin Exp Dermatol 1995;20:436-438.

18 De D, Dogra S, Narang T, Radotra BD, Kanwar AJ: Pityriasis rubra pilaris in a HIV-positive patient (Type 6 PRP). Skinmed 2008;7:47-50.

19 Sertznig P, von Felbert V, Megahed M: Porokeratosis: present concepts. J Eur Acad Dermatol Venereol 2012; 26:404-412.

20 Slominski AT, Zmijewski MA, Skobowiat C, Zbytek B, Slominski RM, Steketee JD: Sensing the environment: regulation of local and global homeostasis by the skin's neuroendocrine system. Adv Anat Embryol Cell Biol 2012;212:v, vii, 1-115. 